\title{
Biodiversity of Drosophila in three different altitudes of Brahmagiri Wildlife Sanctuary, Western Ghats
}

\author{
Koushik Ponnanna C. R. and Krishna M. S.* \\ Drosophila Stock Center, Department Of Studies in Zoology, University of Mysore, Manasagangothri, Mysore 570006, \\ India.
}

Accepted 21 May 2013

\begin{abstract}
Post monsoon studies were conducted to analyze the altitudinal variation in a population of Drosophila (Diptera: Drosophilidae) in Brahmagiri Wildlife Sanctuary belonging to the Western Ghats of Coorg district, Karnataka State, India. A total of 4636 Drosophila flies belonging to 8 species of 3 subgenera were collected at altitudes of 800,900 and $1000 \mathrm{~m}$. The subgenus Sophophora was predominant with 5 different species, subgenus Drosophila with 2 different species and the subgenus Scaptodrosophila was least represented with only a single species. The population density varied in the above three altitudes and highest density was found at $800 \mathrm{~m}$, then the density reduced at $900 \mathrm{~m} \mathrm{(380} \mathrm{flies)}$ and once again the density increased at $1000 \mathrm{~m}$. This shows that Drosophila community is affected by elevation. The diversity of the Drosophila community was also assessed by applying the Simpson and BergerParker indices. At $800 \mathrm{~m}$, the Simpson Index was low at 0.37 and the Berger-Parker index was high at $0.47,900 \mathrm{~m}$. The distributional pattern of a species or related group of species was uneven in space and time. Drosophila kikkawai, Drosophila takahashii, Drosophila immigrans and Drosophila nigra were found in all the three altitudes suggesting that these species are dominant in this region.
\end{abstract}

Key words: Drosophila, Brahmagiri Wildlife Sanctuary, Drosophila kikkawai, Drosophila takahashii, Drosophila immigrans, Drosophila nigra.

\section{INTRODUCTION}

Since the times of Morgan, the species of Drosophila genus forms a very good model organism in all aspects of biological research and by far it forms a better model over bacteria, nematode worm, zebra fish and house mouse. The family Drosophilidae has 3500 described species in various ecosystems throughout the world (Bachli 1998), of which most Genera are concentrated in the tropical regions. The pattern of eco-distribution, biodiversity, Guruprasad et al. (2010) clinal and altitudinal variations (Guruprasad and Hegde, 2006) have been well studied using Drosophila. When compared with other parts of the world, the Drosophila taxonomic studies in India are poorly concentrated as it involves the hardships during work and lack of opportunities in the field. But recently, very few attempts have been made to collect Drosophila from few parts of India Guruprasad et al., (2011). Brahmagiri Wildlife Sanctuary in the Western Ghats of Coorg district is one of such place where collection studies of Drosophila has not been made. This sanctuary derives its name from the highest point, the Brahmagiri peak. The sanctuary has a varying altitude up to $1527 \mathrm{~m}$. The average rainfall in the region is 2500 to 
Table 1. Biodiversity of Drosophila at three different altitudes of Brahmagiri Wildlife Sanctuary during post monsoon season (October to December 2012).

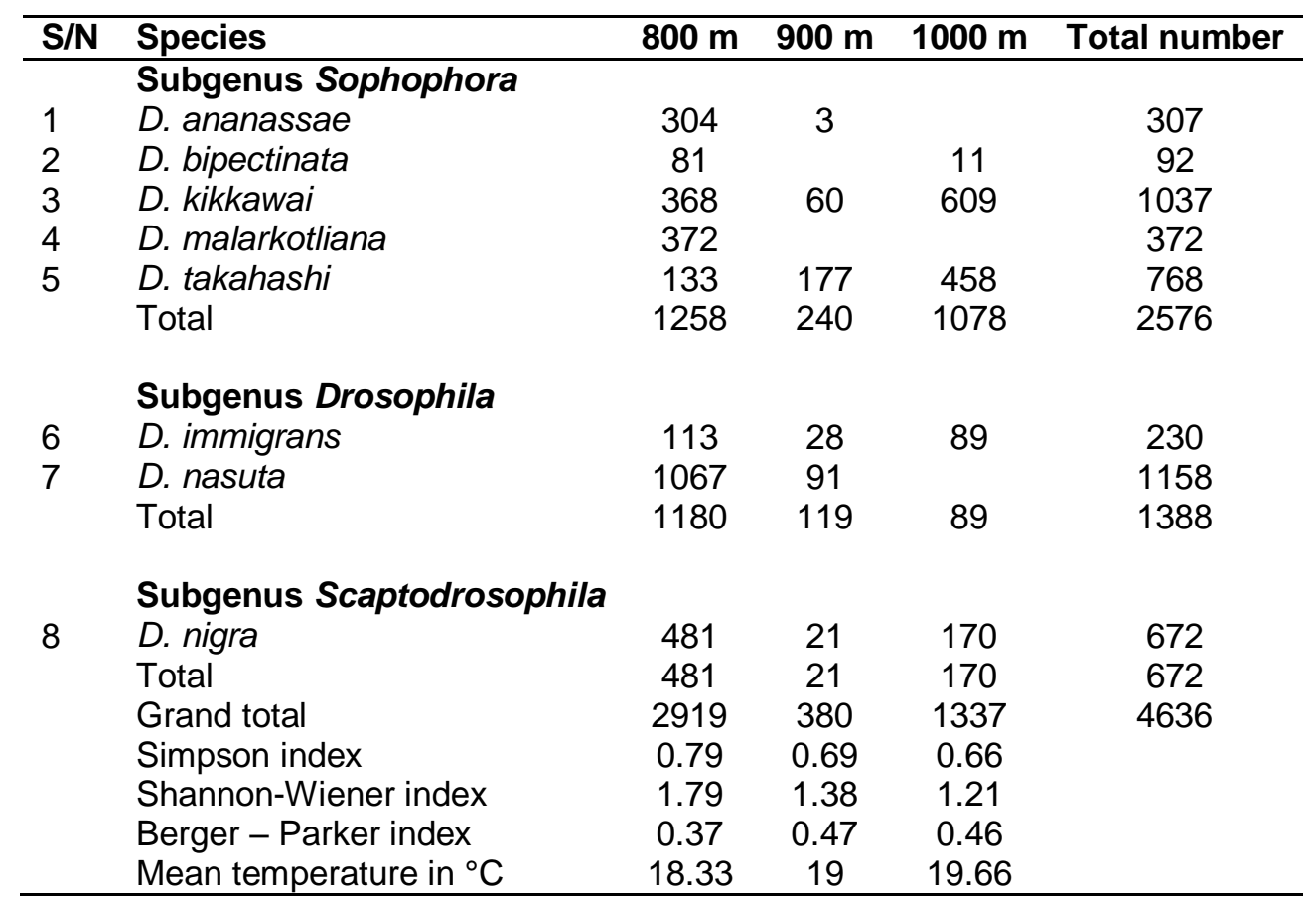

to October). The forests are mainly evergreen and semi evergreen in nature, shola vegetation is dominant in higher altitudes of the sanctuary. Further in the lower regions of the sanctuary, deciduous forests are seen, which are an abode for rich faunal diversity of Drosophilidae. Therefore, the present study has been undertaken in this sanctuary to understand the biodiversity of Drosophila in relation to microhabitat variations.

\section{MATERIALS AND METHODS}

Using sweeping and bottle trapping method, Drosophila collections were made during post monsoon period (October to December 2012) in the Brahmagiri Wildlife Sanctuary in the Western Ghats of Coorg district to account for the biodiversity of Drosophila fauna. The collections were made in three different altitudes $(800,900$ and $1000 \mathrm{~m}$ ) of Brahmagiri Wildlife Sanctuary.

In net sweeping methods, various rotting fruits, such as, Vitas vanifera (grape), Musca paradisca (banana), Solanum lycopersicum (tomato), Manilkara zapota (sapodilla), Citrus sinensis (orange), Malus domestica (apple), Carica papaya (papaya), Citrous auranthium (lime) and Ananas comuses (pineapple), were mixed and spread under shaded areas to attract flies. After a day of spreading, the flies were collected by sweeping using fine net. The flies were then transferred to the bottles containing wheat creamagar medium and brought to laboratory for identification. Bottle trapping method was also followed for collection, in this technique, culturing bottles containing smashed banana sprayed with live yeast were tied to twigs of bushes under shaded areas. The following day, bottles with attracted flies were collected by plugging the bottles and later transferring to culture bottles containing wheat cream-agar medium and brought to the laboratory for identification. The collected males were identified using taxonomical markers such as body pigmentation, sex comb and genital plate. Since there are no such taxonomical markers in females of Drosophila species, therefore the collected females were subjected to isofemale lines. The male flies obtained from the progenies of isofemale lines were used for species identification. Uniformity was maintained in using the techniques and in the number of baits used in the collection sites.

The sanctuary is rich in floral diversity. Vegetation at the collected sites included Acacia catechu, Albizzia amara, Alstonia species, Artocarpus, Bauhinia species, Bombax, Caryota, Calophyllum, Carea, Cinnamomum species, Clementis trifolia, Coffea arabica, Eucalyptus grndis, Ficus bengalensis, Garcinis gummi-gutta, Gymnima sylvestres, Hibiscus malva, Lantana camera, Litsea species, Mesua, Pongamia glabra, Vitex negundo, Holorrhina and Strobilanthes which are the main vegetation growing in the sanctuary.

The abundance, richness and diversity relationship of flies collected were assessed by Simpson (D), Shannon-Wiener $(H)$ and Berger-Parker (1/d) indices (Mateus et al., 2006). The Simpson index (D) that measures the probability that two individuals are randomly selected from a sample that belong to the same species, was calculated using the formula:

$D=\Sigma n(n-1) / N(N-1)$

where, $\mathrm{n}=$ the total number of organisms of a particular species and $\mathrm{N}=$ the total number of organisms of all population.

Shannon-Wiener measures the value of species as a function of their frequency in the community and was calculated using the formula:

$H^{\prime}=-\Sigma p_{i} \ln p_{i}$

$p_{i}=$ the proportion of individuals belonging to the ith species in the 
dataset of interest.

Berger- Parker index (1/d) which shows the relative abundance was calculated using the formula:

$1 / \mathrm{d}=\mathrm{N} / \mathrm{N}_{\operatorname{Max}}$

Where, $\mathrm{N}=$ number of individuals of all species and $\mathrm{Nmax}=$ number of individuals in the most common species.

\section{RESULTS AND DISCUSSION}

The number of flies at three different altitudes of Brahmagiri Wildlife Sanctuary collected during the post monsoon season is shown in Table 1. At $800 \mathrm{~m}$, the number of flies collected was the highest with 2919 flies which comprised of 8 different species. At $900 \mathrm{~m}$, a total of 380 flies belonging to 6 different species were recorded. But at $1000 \mathrm{~m}$, a total of 1337 flies of only 5 different species were collected. Drosophila kikkawai, Drosophila takahashii, Drosophila immigrans and Drosophila nigra species were seen as common species in all altitudes.

The collection data of Drosophila at different altitudes of Brahmagiri Wildlife Sanctuary shows that highest Drosophila density is in the lowest altitude of $800 \mathrm{~m}$, after which it drastically falls at $900 \mathrm{~m}$. Following this, at 1000 $\mathrm{m}$ once again the Drosophila density increased to 1337 flies. This shows that Drosophila community is affected by elevation. Studies by Guruprasad et al. (2011) in Chamundi hill and Wakahama (1962) have also reported the influence of elevation on the distribution of Drosophila flies. They have found that density of Drosophila decreased with increase in elevation. Greater density of Drosophila flies in the lowest altitude found in the present study could be due to the increased floral diversity. The sanctuary is rich in, Maniilkara zapota, Citrus sinensis, Musca paradisca, Garcinis gummi-gutta, Cinnamomum species, Hibiscus malva and at this altitude most of the Coffea arabica and Coffea robusta estates are found. This floral diversity is seen decreasing with increasing altitude.

The value of Simpson, Shannon-Weiner and BergerParker indices that indicate the abundance, richness and diversity of Drosophila flies in different altitudes of the hill are shown in Table 1. At the lowest altitude of $800 \mathrm{~m}$, Simpson $=0.79$; Shannon-Weiner $=1.79$ and BergerParker $=0.37$; at $900 \mathrm{~m}$ Simpson $=0.69$; Shannonweiner $=1.38$ and Berger-Parker $=0.47$. At the higher altitude of $1000 \mathrm{~m}$, Simpson $=0.79$; Shannon-weiner $=$ 1.79 and Berger-Parker $=0.37$

In the Simpson index (D), 0 represents infinite diversity and 1 , no diversity, that is, the greater the value of $D$, the lower the diversity but the reverse is true in the case of Berger-Parker and Shannon-Wiener indices (Ludwig and Reynold, 1988; Mateus et al., 2006). Applying these indices to understand the measures of biodiversity of flies at different altitudes demonstrates that the lower altitude of $800 \mathrm{~m}$ has a higher value (D) and lower value of $1 / \mathrm{d}$ indicating more biodiversity as compared to the higher altitude of 900 and $1000 \mathrm{~m}$ (Table 1).

The density or richness of species also depends on the number of biotic and abiotic factors encountered in the seasons. A change in relative frequency of different species from season to season due to changes in natural environment was reported by Guruprasad et al. (2010). Dobzhansky and Pavan (1950) showed that rainfall appears to have a greater influence on the abundance of Drosophila than temperature. For building larger communities of Drosophila, monsoon is a suitable season and population density declines from the middle of post monsoon due to the prevalence of cold and dry weather (Guruprasad et al., 2011). So, the observed lower densities of Drosophila in the present study can be attributed to the post monsoon climatic conditions. In addition, climatic variables such as humidity, rainfall, temperature and incidence of sun light among others, are determining factors in the occurrence of Drosophila species. These studies suggest that the changes in the natural environment caused by the alteration of seasons would result in the change in relative frequency of different species from season to season.

\section{REFERENCES}

Bachli G (1998). Family Drosophilae. In; L. Papp \& B. Darvas (eds), contributions to a manual of palearctic Diptera. III. Higher Brachteera. Science Herald.

Dobzhansky TH, Pavan C (1950). Local and seasonal variations in relative frequencies of species of Drosophila. Brazilian. J. Anim. Ecol. 19:1-14.

Guruprasad BR, Hegde SN (2006). Altitudinal and seasonal fluctuation of Drosophila fauna of Chamundi hill. Drosophila. inform. Serv. 89:10 11.

Guruprasad BR, Hegde SN, Krishna MS (2010). Seasonal and altitudinal changes in population density of 20 species of Drosophila in Chamundi hill. J. Insect. Sci. 10: 129-139.

Guruprasad BR, Pankaj P (2011). Assesment of Drosophila diversity during monsoon season. J. Entomol. Nematol., 3(4),pp.54-57.

Ludwig JA, Reynolds JF (1988). Statistical ecology. A primer on methods and computing. John Wiley and Sons. p. 337.

Mateus RP, Buschini MLT, Sene FM (2006). The Drosophila community in xerophytic vegetations of the upper Parana- Paraguay River Basin. Brazlian. J . Biol. 66(2): 719-729.

Wakahama KL (1962). Studies on the seasonal variation of population structure in Drosophila, I. Seasonal activity of Drosophilid, flies observed on Mt. Dakesan. Annot. Zoological Japan. 35: 234-242. 